\title{
MECÂNICAS ORTODÔNTICAS E REABSORÇÕES RADICULARES
}

\author{
ORTHODONTIC MECHANICS AND ROOT RESORPTIONS
}

\author{
Antônio Marques de Faria Ganda * \\ Ênio Tonnani Mazzieiro ** \\ Carlos Henrique Theodoro Batista ${ }^{* * *}$
}

\begin{abstract}
RESUMO
A reabsorção radicular externa pode representar uma consequência iatrogênica da terapia ortodôntica, sendo representada pela perda de substância da raiz dentária. Níveis mínimos de perda radicular ortodonticamente induzidos são frequentes e não apresentam significado clínico. Entretanto, quando moderadas ou severas, devem-se adotar condutas baseadas nos fatores de risco e na magnitude das reabsorçôes. Esta revisão de literatura objetiva discutir a relação entre algumas técnicas e mecânicas ortodônticas e seu potencial de causar reabsorçôes radiculares. Observou-se que as causas da reabsorção radicular têm pouca relação com o tipo de mecânica utilizada. Parece existir uma maior relaçáo com o tipo de movimento utilizado, por exemplo, intrusão, do que com o tipo de aparelho empregado para criar as forças. Alguns aspectos das técnicas ortodônticas representam fatores favorecedores de uma maior frequência de reabsorçôes radiculares como, o uso de elásticos intermaxilares, o fechamento de espaço das extraçóes dentárias ortodonticamente indicadas, as mecânicas intrusivas e os deslocamentos dentários extensos.
\end{abstract}

DESCRITORES: Reabsorção da raiz • Ortodontia, mecânica • Etiologia • Fisiopatologia

\section{ABSTRACT}

External apical root resorption is a common iatrogenic consequence of orthodontics therapy and represents root material loss. Minimal level of orthodontically induced root resorption appears to be most frequent and is representative of no clinical significance. Thus, interruption of active treatment can minimize adverse effects during subsequent treatment when loss of root length is moderate or severe. The purpose of this review is to discuss the association of some orthodontic techniques and mechanics and their potential to induce root resorptions. The results suggest that there is little relationship between resorptions and the type of mechanics used. A significantly greater amount of resorption is more common due to type of movement performed, like intrusion, than to the type of appliance designed to create the forces. On the basis of the present investigation, it seems to exist an association between the clinically determined factors of orthodontic therapy and the extent of resorption produced, like wearing intermaxilar elastics, closing orthodontic spaces from extractions, intrusion mechanics and grate amount of tooth movements.

DESCRIPTORS: Root resorption • Orthodontics, mechanics • Etiology • Physiopathology

\footnotetext{
* Especialista e Mestre em Ortodontia pela Pontifícia Universidade Católica (PUC/MG); Professor do Curso de Especialização em Ortodontia da Faculdade de Ciências Médicas e da Saúde de Juiz de Fora (Juiz de Fora/MG); Professor do Curso de Especialização em Ortodontia da Uningá (Ubá/MG). E-mail: antonioganda@gmail.com

** Mestre e Doutor em Ortodontia pela USP (Bauru/SP) e Professor do Curso de Mestrado em Ortodontia da Pontifícia Universidade Católica (PUC/MG). E-mail: mazzieiro@pucminas.br

*** Especialista em Disfunção Têmporo-Mandibular e Dor Orofacial pela Faculdade de Odontologia da Universidade Federal de Juiz de Fora (FO/UFJF/Juiz de Fora/MG); Prof. Convidado do Curso de Especialização em Disfunção Têmporo-Mandibular e Dor Orofacial da Pontifícia Universidade Católica (PUC/ MG); Aluno do Curso de Especialização em Ortodontia da Faculdade de Ciências Médicas e da Saúde de Juiz de Fora (Juiz de Fora/MG). E-mail: clinicaatm@carloshenrique.odo.br
} 


\section{INTRODUÇÃO}

Todo o tratamento ortodôntico, seja qual for a mecânica utilizada, está sujeito a intercorrências que podem levar danos aos dentes e a suas estruturas de suporte. As reabsorçóes radiculares representam um dos mais frequentes processos iatrogênicos ortodonticamente provocados.

As reabsorçóes radiculares, termo utilizado para de- signar a perda de substância da raiz dentária, podem advir de uma variedade de fatores como traumas, lesóes periapicais infecciosas, doenças periodontais, além de outros fatores desconhecidos. As reabsorções causadas pela movimentação ortodôntica, apesar de apresentarem o mesmo aspecto, representam um outro tipo de processo. Alguns autores as definem como reabsorçôes radiculares inflamatórias induzidas ortodonticamente, pois se
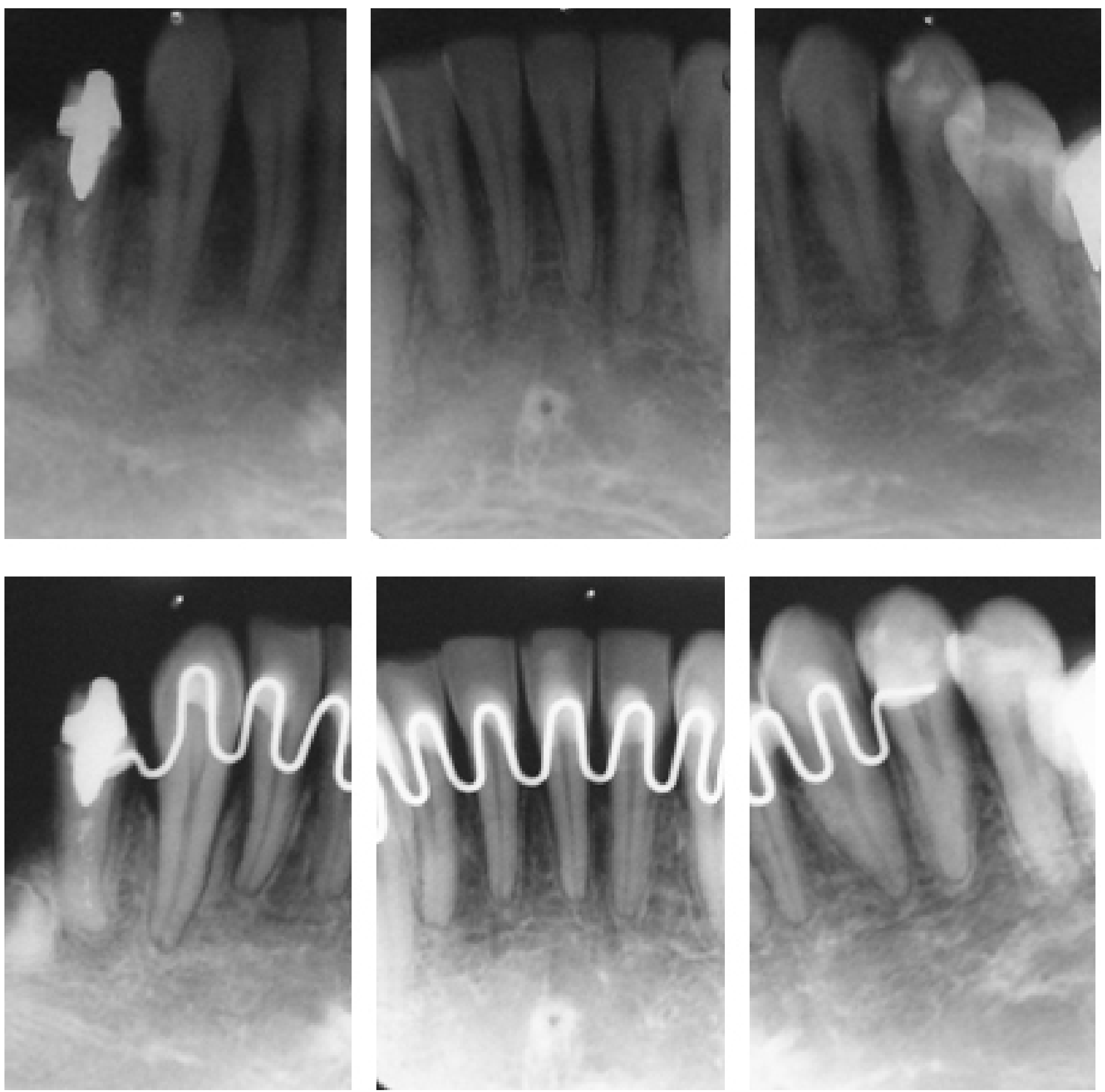

Figura 1 - Radiografias periapicais ilustrando reabsorção radicular leve. Incisivos inferiores $(A, B, C)$ antes e $(D, E, F)$ depois do tratamento ortodôntico. 
caracterizam por processos inflamatórios assépticos extremamente complexos (Brezniak e Wasserstein', 2002), podendo levar a uma necrose isquêmica no ligamento periodontal (Pizzo et al. $\left.{ }^{35}, 2007\right)$.

A quantidade e severidade das reabsorçôes variam muito. A perda de material radicular pode atingir o cemento, a dentina e, em casos mais graves, até mesmo o tecido pulpar. No caso de acometimento da dentina, o processo torna-se irreversível. Estudos microscópicos revelam uma alta incidência de reabsorçôes radiculares por permitirem a visualização de todas as faces da raiz, além de revelarem lesões invisíveis macroscopicamente. Por outro lado, estudos radiográficos revelam incidência menos elevada, pois permitem somente a análise de reabsorçóes apicais e, mais raramente, reabsorçôes proximais. As reabsorçóes severas comprometem o sucesso do tratamento ortodôntico. Entretanto, a maioria das reabsorçóes provocadas pela Ortodontia não compromete a longevidade e funcionalidade dos dentes envolvidos (Brezniak e Wasserstein ${ }^{8}$, 1993).

Esta revisão de literatura tem o objetivo de discutir a relação entre algumas técnicas e mecânicas ortodônticas e seu potencial de causar reabsorçóes radiculares.

\section{REVISÃO DA LITERATURA}

Técnica Edgewise

A maioria dos estudos sobre reabsorção radicular direciona-se aos incisivos, especialmente os superiores, pois são os dentes mais acometidos pelo processo durante o tratamento ortodôntico (L'Abee e Sanderink ${ }^{24}$, 1985; Parker e Harris ${ }^{34}$, 1998).

Segundo Deshields ${ }^{15}$ (1969), 99\% dos casos tratados ortodonticamente apresentam reabsorção radicular em pelo menos um incisivo superior. De uma maneira geral, os incisivos laterais são mais acometidos por reabsorções radiculares que os incisivos centrais. Freitas et al. ${ }^{17}$ (2007) averiguaram que há uma correlação estatisticamente significante entre a correção do overjet e a retração de incisivos superiores com o grau de reabsorção. Assim Mohandesan et al. ${ }^{32}$ (2007) sugeriram que deveria haver cautela com pacientes cuja mecânica necessita de extração de pré-molares.

As reabsorções são classificadas em leves (Figura 1) ou moderadas (Figura 2), variando de um contorno irregular do ápice radicular até um terço de raiz reabsorvida (Deshields ${ }^{15}$, 1969; Lupi et al. ${ }^{28}$, 1996). As reabsorçóes severas, caracterizadas por mais de um terço de raiz reabsorvida (Figura 3),
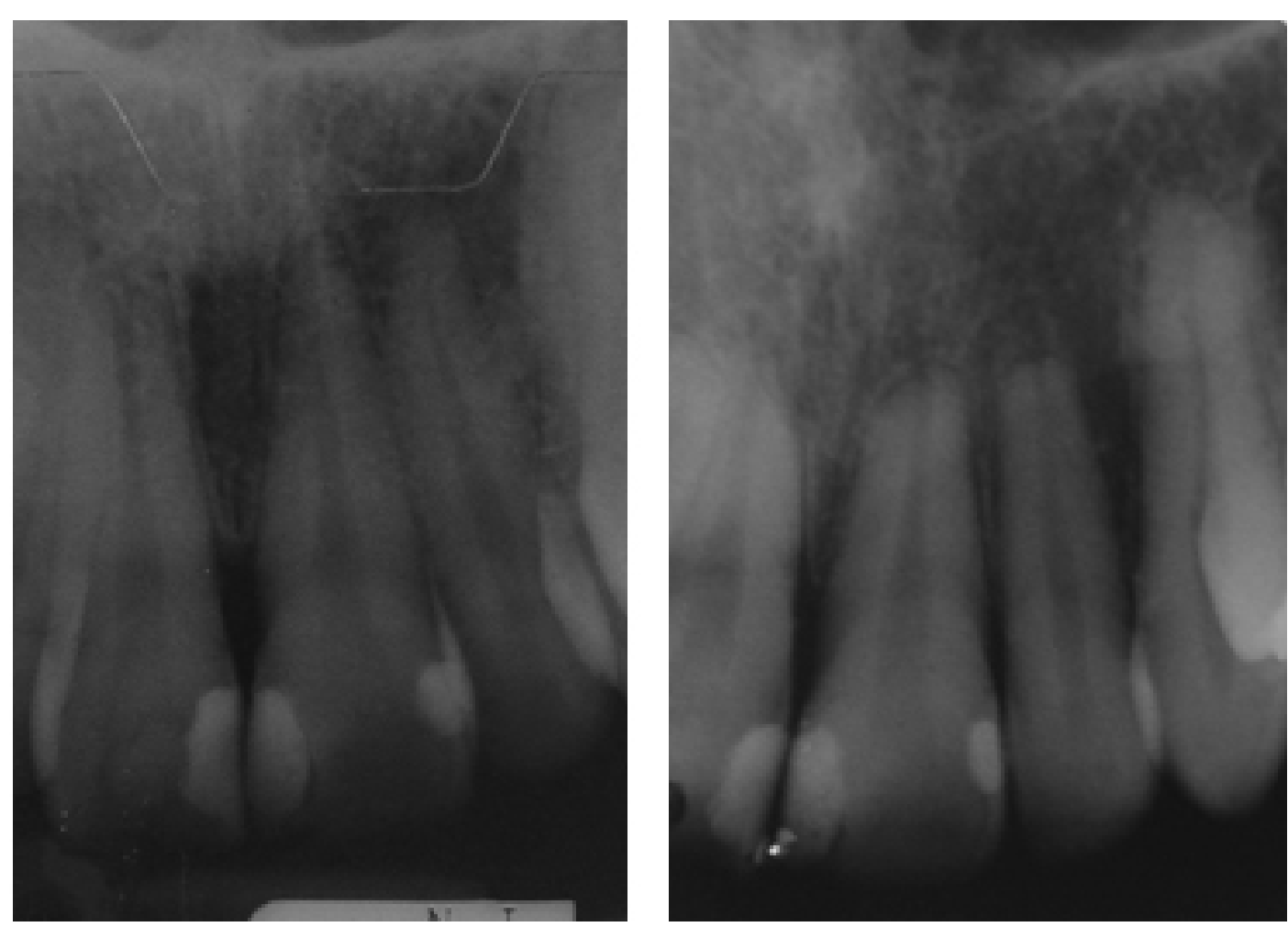

Figura 2 - Radiografias periapicais ilustrando reabsorção radicular moderada. Incisivos superiores (A) antes $e(B)$ depois do tratamento ortodôntico. são menos frequentes e apresentam uma incidência de aproximadamente 2 a 3\% dos incisivos superiores submetidos ao tratamento ortodôntico (Deshields ${ }^{15}$, 1969; Kaley e Phillips ${ }^{22}$, 1991; Lupi et al. 28 , 1996). Linge e Linge $^{27}$ (1991) encontraram uma reabsorção radicular média de $0,9 \mathrm{~mm}$ para cada incisivo superior, sendo que $16,5 \%$ dos pacientes apresentaram valores maiores que $2,5 \mathrm{~mm}$ para um ou mais incisivos superiores. 
Com relação aos incisivos centrais superiores mais especificamente, Taner et al. ${ }^{40}$ (1999) afirmam que há uma menor incidência de reabsorçōes em indivíduos Classe I que nos indivíduos Classe II. Copeland e Green ${ }^{12}$ (1986) também encontraram resultados similares, ao verificarem uma quantidade média de reabsorção de $2,93 \mathrm{~mm}$ durante o tratamento ativo. Entretanto, durante o período de contenção a média foi de $0,1 \mathrm{~mm}$, indicando que, com o término do tratamento ativo, a reabsorçáo apical da raiz se paralisaria.

Os incisivos inferiores apresentam um índice bem menor de reabsorção radicular se comparados aos superiores. Segundo Kaley e Phillips ${ }^{22}$ (1991) aproximadamente $40 \%$ dos pacientes apresentaram no mínimo reabsorção moderada em pelo menos um dos incisivos superiores e $20 \%$ em ambos os incisivos centrais inferiores. Entretanto, Lupi $e t$ al. ${ }^{28}$ (1996) afirmam não terem encontrado diferenças estatisticamente significativas entre os dois grupos de dentes.

Com relação aos segundos pré-molares, Kaley e Phillips $^{22}$ (1991) observaram que apenas 6\% dos pacientes apresentam reabsorção em pelo menos um elemento desse grupo de dentes.

\section{Técnica de Begg}

Gaudet $^{18}$ (1970) realizou um estudo experimental microscópico em macacas no qual ele avaliou as reaçóes histológicas em incisivos superiores submetidos a contro-
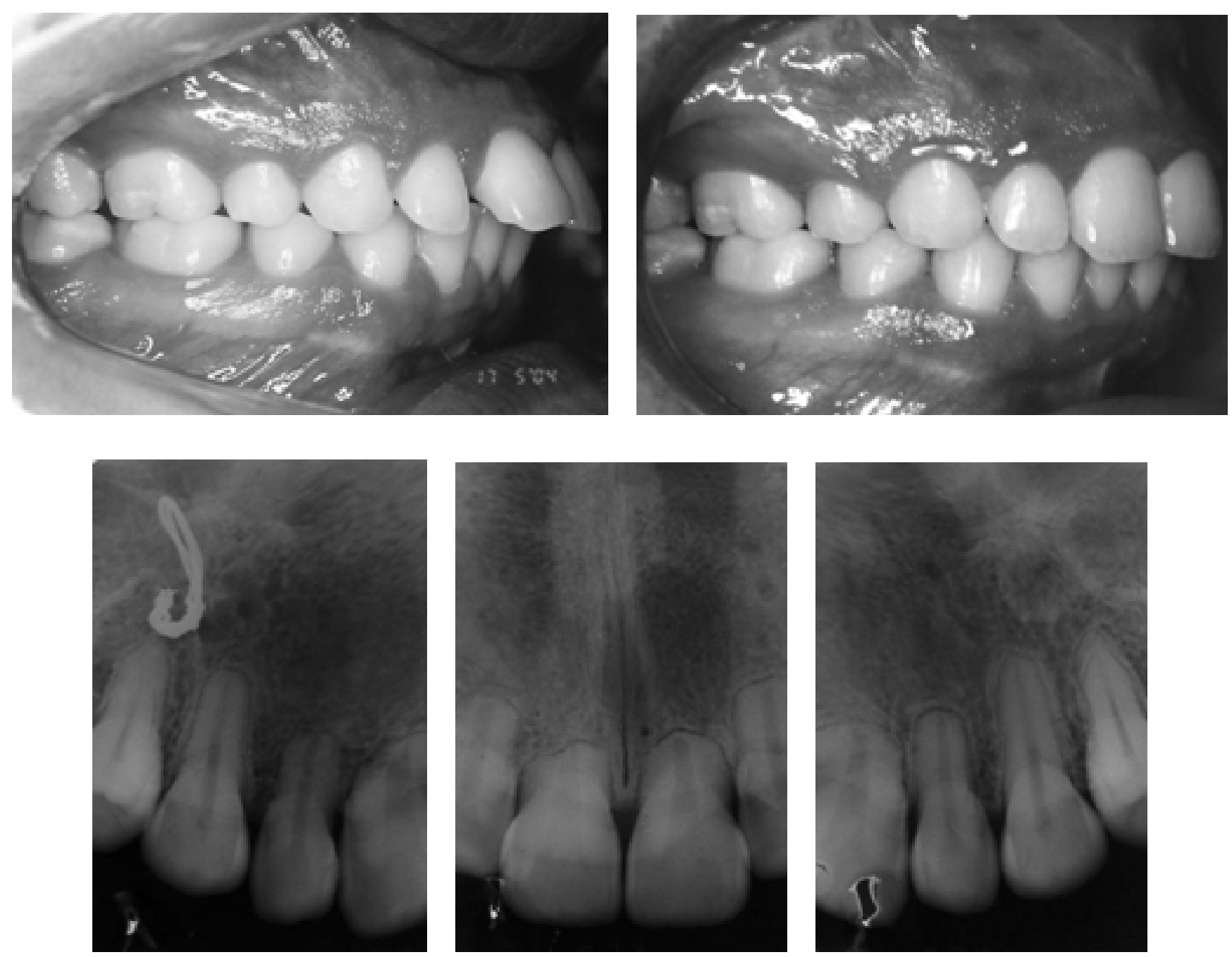

Figura 3 - Fotografias de um tratamento de má oclusão de Classe II divisão 1 com exodontias de primeiros pré-molares superiores: antes do tratamento (A) e depois do tratamento (B). Radiografias ilustrando reabsorção radicular severa após o tratamento (C, $D$, E). 
le de torque lingual com mecânica de Begg e constatou que os dentes submetidos a maior carga mostraram um maior número de reabsorçóes radiculares, com comprometimento dentinário variando de leve a severo. O dente submetido à menor força apresentou pouca reabsorção radicular, sem comprometimento dentinário.

Segundo L'Abee e Sanderink ${ }^{24}$ (1985), o efeito da técnica de Begg sobre as raízes de incisivos centrais quanto às reabsorções radiculares é significativamente maior no primeiro estágio da técnica e menor no segundo. Não se observou diferença significante entre o primeiro e o terceiro estágios da terapia. Um outro dado importante é a presença de reabsorçóes sempre que os incisivos são movimentados contra a cortical palatina (Ten Hoeve e Mulie $\left.^{41}, 1976\right)$, sendo que as lesôes mais frequentemente observadas se estendem do ápice à face palatina da raiz (Ten Hoeve e Mulie ${ }^{41}$, 1976).

Rosenberg $^{36}$ (1972) avaliou a incidência e a quantidade de reabsorção radicular apical de caninos e segundos pré-molares inferiores e superiores com rizogênese incompleta por meio de radiografias periapicais. Trinta e sete por cento dos dentes avaliados sofreram reabsorção radicular, com valores médios para cada dente estatisticamente insignificantes (menores que $0,5 \mathrm{~mm}$ ). Apenas $6 \%$ apresentaram reabsorçóes maiores que $2 \mathrm{~mm}$. A reabsorção nos caninos foi significativamente maior que nos pré-molares.

\section{Comparação entre diferentes técnicas}

Barbagallo et al. ${ }^{5}$ (2008) utilizaram a microtomografia para quantificar a reabsorção radicular produzida por tratamento ortodôntico realizado com aparelhos termoplásticos sequenciais removíveis e compararam com forças ortodônticas convencionais leves e pesadas. Portanto, puderam concluir que tais aparelhos têm efeitos de reabsorção no cemento radicular similares aos dos aparelhos ortodônticos fixos quando com forças leves de $25 \mathrm{~g}$.

$\mathrm{McNab}$ et al. ${ }^{31}$ (2000) compararam radiograficamente as reabsorçóes radiculares provocadas em dentes posteriores pelas técnicas de Begg e Edgewise, notando uma incidência de reabsorção 2,3 vezes maior para os pacientes tratados pela técnica de Begg. Em relação à extração de dentes, para as duas técnicas, a ocorrência de reabsorção é de aproximadamente 3,72 vezes maior para tratamentos com extraçóes. Por outro lado, Beck e Harris $^{6}$ (1994) não encontraram diferença estatisticamente significante entre as duas técnicas. Este achado negativo sugere que as causas da reabsorçáo radicular têm pouca relação com o tipo de mecânica utilizada. Parece existir uma maior relação com o tipo de movimento utilizado, por exemplo, intrusão, do que com o tipo de aparelho utilizado para criar as forças. Os dentes mais frequentemente afetados foram os incisivos centrais superiores e os menos afetados os primeiros molares inferiores, mais precisamente a raiz distal.

Mavragani et al. ${ }^{29}$ (2000) compararam a extensão da reabsorção do ápice radicular em pacientes tratados pelas mecânicas de Edgewise e Straight-wire. Todos os pacientes apresentavam Classe II divisão 1 e todos os tratamentos foram feitos com extraçóes de pré-molares. Os incisivos centrais do grupo Edgewise apresentaram reabsorçóes apicais significativamente maiores que os do grupo Straight-wire. Não se observou diferença significativa para os incisivos laterais. Também não se encontraram diferenças no grau de reabsorçâo entre os incisivos centrais e laterais do mesmo paciente. Silva Filho et al..$^{39}$ (1993) observaram que, para ambas as técnicas, todos os dentes avaliados sofreram um encurtamento radicular, não existindo diferença estatisticamente significativa entre as técnicas, resultado semelhante ao encontrado por Mohandesan et al. ${ }^{32}$ (2007) quando compararam as duas técnicas. Os incisivos superiores sofreram maior reabsorção, seguidos pelos incisivos inferiores, molares, caninos e pré-molares, respectivamente (Silva Filho et al. 39 , 1993). De qualquer forma é relevante destacar que o tratamento da má oclusão de Classe II divisão 1 com extração de pré-molares sugere necessitar de retração dos dentes anteriores, o que tende a promover um movimento radicular através da cortical palatina da maxila e isso sugere contribuir para o processo de reabsorção radicular (Remmelink e Van Der Molen ${ }^{19}$, 1984).

Mohandesan et al. ${ }^{32}$ (2007) encontraram reabsorção clinicamente significante em $74 \%$ dos incisivos centrais e $82 \%$ dos incisivos laterais. Diante desse achado, os autores sugeriram que o monitoramento radiográfico metódico dos incisivos superiores em intervalos frequentes poderia auxiliar no diagnóstico de reabsorçôes e na identificação daqueles que poderiam tornar-se severamente afetados, sugestáo também adotada por Pizzo et al. ${ }^{35}$ (2007). Abuabara ${ }^{1}$ (2007) reforça que em casos rotineiros deve-se solicitar o exame radiográfico 6 meses após o início do tratamento e em casos de maior risco, a cada 3 meses.

Janson et al. ${ }^{21}$ (2000) compararam radiograficamente a quantidade de reabsorção radicular após o tratamento ortodôntico de pacientes com as técnicas Edgewise, Strai- 
ght-wire e Terapia Bioeficiente. Os autores encontraram uma similaridade na quantidade de reabsorção para as técnicas Edgewise e Straight-wire, porém menor para a Terapia Bioeficiente.

Parker e Harris ${ }^{34}$ (1998) compararam adolescentes tratados pelas técnicas Edgewise, Begg e Roth, sendo que todos os casos foram conduzidos com extraçáo de quatro pré-molares para aliviar apinhamento ou protrusão anterior. Não se observaram diferenças estatisticamente significativas entre as três técnicas com relação à reabsorção das raízes, nem em relação aos sexos. Durante todo o tratamento ativo, encontrou-se uma reabsorção média para a amostra total de $1,4 \mathrm{~mm}$.

Blake et al. ${ }^{7}$ (1995) compararam a reabsorção radicular provocada pela técnica Edgewise e pelo Speed Applian$c e$, não observando diferença estatisticamente significativa para os valores de reabsorção radicular entre as duas técnicas. Nos casos com extração, para ambas as técnicas, notou-se uma incidência estatisticamente maior de reabsorção nos incisivos laterais superiores que nos casos sem extração.

\section{Técnica Bioprogressiva}

McFadden et al..$^{30}$ (1989) relatam que a reabsorção radicular resultante da intrusão de incisivos superiores e inferiores com a técnica bioprogressiva apresenta um valor médio de $1,84 \mathrm{~mm}$ de reabsorção nos incisivos superiores e $0,61 \mathrm{~mm}$ nos inferiores, correspondendo a uma redução de 13,2 e 4,3\% do comprimento radicular, respectivamente. Entretanto, não se observou uma correlação estatisticamente significante entre a quantidade de reabsorção e a de intrusão dos incisivos, nem entre os casos com e sem extração.

\section{Mecânica de intrusão}

Costopoulos e Nanda ${ }^{13}$ (1996) avaliaram radiograficamente a relação entre a mecânica de intrusão de Burstone e as reabsorçóes radiculares em incisivos superiores e encontraram uma quantidade média de reabsorção radicular de 0,6 mm para os incisivos centrais. Entretanto, Dermaut e Munck ${ }^{14}$ (1986) observaram uma reabsorção média de $2,5 \mathrm{~mm}$ das raízes (18\% do comprimento radicular), não sendo observada diferença estatisticamente significante entre as reabsorçóes nos incisivos centrais e laterais.

Chiqueto et al. ${ }^{10}$ (2008) também avaliaram radiograficamente a influência da mecânica de intrusáo em incisivos, porém a mecânica foi realizada por meio de curva de Spee acentuada ou reversa em uma amostra de 60 pacientes. $\mathrm{O}$ grupo com mordida profunda teve mais reabsorção radicular estatisticamente significante do que o grupo com overbite normal. Concluíram que a acentuação ou reversão da curva de Spee nos arcos causam mais reabsorção do que mecânicas não intrusivas.

\section{Mecânicas de retração: deslize $x$ alças seccionais}

Alexander ${ }^{3}$ (1996) relatou que em pacientes Classe I, com apinhamento anterior e tratados com extração dos quatro primeiros pré-molares, não se observou diferença estatisticamente significativa entre as técnicas com arco contínuo (mecânica de deslize) e com alças seccionais de retração (fio 0,017"x0,025" de TMA), ambas com aparelho pré-ajustado prescrição Roth.

\section{Forças continuas $x$ forças descontinuas}

Acar et al. ${ }^{2}$ (1999) estudaram microscopicamente o efeito da aplicação de forças contínuas e descontínuas em relação à reabsorção radicular. Para os dois tipos de forças, notou-se a formação de cavidades de reabsorção na superfície vestibular das raízes. Tais cavidades mostravam-se mais amplas no terço médio e cervical. Percebeu-se um comprometimento da dentina na maioria das lesôes. A área de reabsorção da superfície vestibular das raízes foi de $11 \%$ para as forças contínuas e $6 \%$ para as descontínuas. Observou-se também reabsorção no terço apical da superfície lingual das raízes (zona de pressão), significativamente maior no grupo de forças contínuas.

Num sistema de forças interrompidas, sem ativaçóes semanais e com períodos de repouso, pode-se esperar menor estresse tissular e possivelmente um movimento dentário mais linear com menor reabsorção radicular (Viazis $\left.{ }^{42}, 1995\right)$.

Kurol et al. ${ }^{23}$ (1996) estudaram radiograficamente as reabsorçôes radiculares em pré-molares superiores submetidos a uma força ortodôntica contínua e observaram que $92,9 \%$ dos dentes apresentaram reabsorção radicular. Entretanto, esses resultados vão de encontro aos de Owman-Moll et al. ${ }^{33}$ (1995) que, apesar de terem encontrado áreas de reabsorção em todos os dentes avaliados, não observaram diferenças no número e severidade das áreas reabsorvidas entre os dois grupos experimentais.

\section{Elásticos intermaxilares}

Segundo Linge e Linge ${ }^{27}$ (1991), um aumento significativo na quantidade de reabsorção radicular ocorreu 
nos casos em que se usaram elásticos. Os autores também sugerem que as forças de vai-e-vem provenientes do uso de elásticos provocam aumento das reabsorçóes radiculares em incisivos. Tais achados apóiam aqueles de Mavragani et al. ${ }^{29}$ (2000) que encontraram uma correlação significativamente positiva entre as reabsorções radiculares dos incisivos centrais e a duração do uso de elásticos de Classe II.

Por outro lado, Deshields ${ }^{15}$ (1969) encontrou uma correlação positiva apenas quando se usaram os elásticos de Classe II em combinação com aparelho extrabucal. Quando se consideraram os elásticos de Classe II e os aparelhos extrabucais separadamente, não se observou correlação significativa com as reabsorçóes radiculares.

Rudolph $^{37}$ (1940) relatou que os elásticos de Classe III, usados para o preparo de ancoragem, aumentam a reabsorção radicular na raiz distal do primeiro molar superior.

\section{Aparelho Extrabucal}

Segundo Hixon et al. ${ }^{20}$ (1969), a tração cervical causa pouca reabsorção nas raízes dos molares. Entretanto, têm-se negligenciado muito as sequelas do tratamento ortodôntico com distalização dos molares.

Langford e Sims ${ }^{26}$ (1981) reportaram um caso clínico no qual uma paciente usou um aparelho extrabucal de tração cervical para distalização dos primeiros molares superiores. $\mathrm{O}$ aparelho foi usado por 5 meses e meio. Dezenove meses depois, decidiu-se extrair tais dentes e não os primeiros pré-molares superiores, como planejado anteriormente, devido à severa reabsorção radicular constatada radiograficamente. Os primeiros molares superiores foram analisados microscopicamente e observaram-se extensas reabsorçóes na face distal de todas as raízes, sendo a raiz distovestibular do primeiro molar superior esquerdo completamente reabsorvida. Tal reabsorção teria ocorrido devido ao contato da raiz do primeiro molar com o germe do segundo molar.

\section{Expansão Rápida da Maxila (ERM)}

Segundo Consolaro ${ }^{11}$ (2002), a reabsorção radicular na ERM ocorre na face vestibular das raízes dos pré-molares em áreas variando entre 2340 a $3200 \mu \mathrm{m}$ e profundidade de 390 a $1600 \mu \mathrm{m}$. Os pré-molares funcionam como pontos de ancoragem para que ocorra a abertura da sutura palatina mediana. Apesar de extensas, as reabsorçóes radiculares resultantes da ERM mostram-se rasas e não comprometem a polpa dentária ou a resistência da estrutura radicular.

Silva Filho et al. ${ }^{38}$ (1994) realizaram um estudo para diagnosticar radiograficamente possíveis alteraçóes ocorridas nas estruturas periodontais e dentárias dos pré-molares que serviram de ancoragem para o aparelho expansor de Haas (ancorado nos primeiros pré-molares e nos primeiros molares superiores). Após um período ativo de tratamento e um período de aproximadamente 3 a 4 meses de contenção, removiam-se os aparelhos, podendo-se constatar, por meio de radiografias periapicais, a ausência de imagens sugestivas de reabsorção radicular proximal ou arredondamento de ápice radicular dos dentes avaliados. Porém, radiograficamente, a superposição de imagens nas películas periapicais dificilmente permitirá o delineamento das áreas de reabsorção radicular, se elas ocorrerem na superfície vestibular dos pré-molares conforme afirmou Consolaro ${ }^{11}$ (2002). Observou-se também a não interrupção do processo de rizogênese nos pacientes com os ápices radiculares incompletos.

Nesse sentido, Langford ${ }^{25}$ (1982) estudou microscopicamente os efeitos da ERM em pacientes com indicação ortodôntica de extração dos primeiros pré-molares superiores após a remoção do disjuntor de Haas. Observaram-se grandes áreas de reabsorção, principalmente na face vestibular da raiz. As áreas reabsorvidas pareceram mais lateralmente expansivas que axialmente invasivas, de maneira que as áreas envolvidas apresentavam-se largas, mas a integridade pulpar preservada. Encontraramse reabsorçôes mínimas na superfície lingual dos prémolares. Utilizando-se de uma metodologia semelhante com microscópio, Barber e Sims ${ }^{4}$ (1981) encontraram cerca de $36 \%$ das faces vestibulares das raízes dos prémolares acometidas por reabsorção radicular, sendo que o processo continuou mesmo após o término do período ativo de tratamento. Langford ${ }^{25}$ (1982) concluíram também que as principais reabsorçóes ocorrem até os três primeiros meses de contenção fixa.

Everdi et al. ${ }^{16}$ (1994) avaliaram os primeiros pré-molares superiores com indicação ortodôntica de extração de pacientes submetidos à ERM, com aparelho expansor de Haas e com expansor encapsulado dentossuportado. Todos os dentes apresentaram áreas de reabsorção de cemento ou de cemento e dentina. A maioria das cavidades encontrava-se na superfície vestibular das raízes, com poucas na região apical. Nenhuma diferença expressiva foi notada quanto à natureza ou frequência das lesões entre os dois métodos de expansão. Entretanto, com relação à localização, nos pacientes tratados com expansor 
de Haas, as lesões encontravam-se frequentemente na região vestibular, enquanto que em pacientes tratados com expansor encapsulado, as lesóes se localizavam nas regiôes vestibular, apical e mesial.

\section{CONCLUSÃO}

A literatura acerca das reabsorçóes radiculares ortodonticamente provocadas deixa clara a ideia de que o processo representa uma iatrogenia presente na grande maioria dos tratamentos, independente da técnica utilizada.

Relacionar as diferentes técnicas ortodônticas com seu potencial de causar as reabsorçôes radiculares tornase uma tarefa complexa. A dificuldade de se avaliarem precisa e comparativamente as várias técnicas, quanto ao índice de reabsorção resultante, decorre da falta de padronização e rigor na elaboração das amostras e das casuísticas. Nos trabalhos avaliados, alguns questionamentos permanecem sem resposta, como o tipo de má oclusão inicial, o padrão de diagnóstico e o planejamento, a padronização de radiografias, a calibração no tratamento, o grau de experiência do ortodontista, os critérios para a classificação e para o diagnóstico das reabsorçóes radi- culares.

O início e a progressão da reabsorção radicular estão associados com fatores de risco relacionados ao tratamento ortodôntico como, por exemplo, o uso de elásticos intermaxilares, o fechamento de espaço das extraçóes dentárias ortodonticamente indicadas, as mecânicas intrusivas, os deslocamentos dentários extensos, além de outros como a duraçáo do tratamento, magnitude das forças empregadas, a direção do movimento dentário, torque e método de aplicação das forças (contínuas versus intermitentes). Os fatores de risco inerentes ao paciente são susceptibilidade genética, algumas doenças sistêmicas (alterações metabólicas de cálcio e fósforo), anormalidades na morfologia radicular, trauma dental, uso de medicamentos e dentes endodonticamente tratados.

O que se pode observar é o fato de que o tipo de técnica ortodôntica empregada no tratamento não apresenta uma ligação direta com o processo de perda de substância radicular. $\mathrm{O}$ processo parece estar mais relacionado às características das forças empregadas, tais como intensidade, direção, frequência e duração. Outro fator que também apresentam alguma influência é o tipo de movimento dentário, bem como a sua extensão.

\section{REFERÊNCIAS}

1. Abuabara A. Biomechanical aspects of external root resorption in orthodontic therapy. Med Oral Patol Oral Cir Bucal 2007 Dec; 12(8):610-13.

2. Acar A, Canyurek U, Kocaaga M, Erverd N. Continuous vs. discontinuous force application and root resorption, Angle Orthod 1999 Apr; 69(2): 159-63.

3. Alexander SA. Levels of root resorption associated with continuous arch and sectional arch mechanics. Am J Orthod Dentofacial Orthop 1996 Sep; 110(3): $321-4$.

4. Barber AF, Sims MR. Rapid maxillary expansion and external root resorption in man: a scanning electron microscope study. Am J Orthod 1981 Jun; 79(6): 63052.
5. Barbagallo LJ, Jones AS, Petocz P, Darendeliler MA. Physical properties of root cementum: Part 10. Comparison of the effects of invisible removable thermoplastic appliances with light and heavy orthodontic forces on premolar cementum. A microcomputed-tomography study. Am J Orthod Dentofacial Orthop 2008 Feb; 133(2): 218-27.

6. Beck BW, Harris EF. Apical root resorption in orthodontically treated subjects: analysis of Edgewise and light wire mechanics. Am J Orthod Dentofacial Orthop 1994 Apr; 105(4):350-61.

7. Blake M, Woodside DG, Pharoah MJ. A radiographic comparison of apical root resorption after orthodontic treatment with Edgewise and speed appliances. Am J Orthod Dentofacial Orthop 1995 Jul; 108(1): 76-84.

8. Brezniak N, Wasserstein A. Root resorption after orthodontic treatment: part I - Literature review. $A m$ J Orthod Dentofacial Orthop 1993 Jan; 103(1):62-6. 
9. Brezniak N, Wasserstein A. Orthodontically induced inflammatory root resorption.Part II: the clinical aspects. Angle Orthod 2002 Apr; 72(2): 180-4.

10. Chiqueto K, Martins DR, Janson G. Effects of accentuated and reversed curve of Spee on apical root resorption. Am J Orthod Dentofacial Orthop 2008 Feb; 133(2):261-8 quiz 328. e2.

11. Consolaro A. Reabsorções dentárias na movimentação ortodôntica. In: Consolaro A. Reabsorções radiculares nas especialidades clínicas. Maringá: Dental Press; 2002.

12. Copeland S, Green LJ. Root resorption in maxillary incisor following active orthodontic treatment. $A m$ J Orthod 1986 Jan; 89(1):51-5.

13. Costopoulos G, Nanda R. An evaluation of root resorption incident to orthodontic intrusion. $A m \mathrm{~J}$ Orthod Dentofacial Orthop, 1996 May; 109(5): 543-8.

14. Dermaut LR, Munck A. Apical root resorption of upper incisor caused by intrusive tooth movement: a radiographic study. Am J Orthod Dentofacial Orthop 1986 Oct; 90(4): 321-6.

15. Deshields RW. A study of root resorption in treated class II, division I malocclusion. Angle Orthod 1969 Oct; 39(4): 231-45.

16. Erverdi N, Okar I, Kucukkeles N, Arbak S. A comparison of two different rapid palatal expansion techniques from the point of root resorption. Am J Orthod Dentofacial Orthop 1994 Jul; 106(1): 47-51.

17. Freitas MR, Beltrão RT, Janson G, Henriques JF, Chiqueto K. Evaluation of root resorption after open bite treatment with and without extractions. Am J Orthod Dentofacial Orthop 2007 Aug; 1329(2): 143 e 15-22.

18. Gaudet EL. Tissue changes in monkeys following root torque with Begg technique. Am J Orthod 1970 Aug; 58(2): 167-78.

19. Remmelink HJ, Van Der Molen AL. The effect of anteroposterior incisor repositioning on the root and cortical plate: a follow-up study. J Clin Orthod 1984 Jan; 18(1):42-9.
20. Hixon EH, Atikian H, Callow GE, McDonald HW, Tacy RJ. Optimal force, differential force, and anchorage. Am J Orthod 1969 May; 55(5): 437-57.

21. Janson GR, De Luca Canto G, Martins DR, HENriques JF, De Freitas MR. A radiographic comparison of apical root resorption after orthodontic treatment with 3 different fixed appliance techniques. Am J Orthod Dentofacial Orthop 2000 Sep; 118(3): 262 73.

22. Kaley J, Phillips C. Factors related to root resorption in Edgewise practice. Angle Orthod 1991; 61(2): 12532.

23. Kurol J, Owman-Moll P, Lundgren D. Time-related root resorption after application of a controlled continuous orthodontic force. Am J Orthod Dentofac ial Orthop 1996 Sep; 110(3):303-10.

24. L'Abee EM, Sanderink GC. Apical root resorption during Begg treatment. J Clin Orthod 1985 Jan; 19(1):60-1.

25. Langford SR. Root resorption extremes resulting from clinical RME. Am J Orthod 1982 May; 81(5): $371-7$.

26. Langford SR, Sims MR. Upper molar root resorption because of distal movement. Report of a case. Am J Orthod Dentofacial Orthop 1981 Jun; 79(6):66979.

27. Linge L, Linge BO. Patient characteristics and treatment variables associated with apical root resorption during orthodontic treatment. Am J Orthod Dentofacial Orthop 1991 Jan; 99(1): 35-43.

28. Lupi JE, Handelman CS, Sadowsky C. Prevalence and severity of apical root resorption and alveolar bone loss orthodontically treated adults. Am J Orthod Dentofacial Orthop 1996 Jan; 109(1): 28-37.

29. Mavragani M, Vergari A, Selliseth NJ, Bøe OE, Wisth PL. Radiographic comparison of apical root resorption after orthodontic treatment with a standard Edgewise and a Straight-wire Edgewise technique. Eur J Orthod 2000 Dec; 22(6):665-74.

30. Mcfadden WM, Engstrom C, Engstrom H, Anholm JM. A study of the relationship between incisor intrusion and root shortening. Am J Orthod Dentofacial Orthop 1989 Nov; 96(5): 390-6. 
31. McNab S, Battistutta D, Taverne A, Symons AL. External apical root resorption following orthodontic treatment. Angle Orthod 2000 Jun; 70(3):227-32.

32. Mohandesan H, Ravanmehr H, Valaei N. A radiographic analysis of external apical root resorption of maxillary incisors during active orthodontic treatment. Eur J Orthod 2007 Apr; 29(2): 134-39.

33. Owman-Moll P, Kurol J, Lundgren D. Continuous versus interrupted continuous force related to the tooth movement and root resorption. Angle Orthod 1995; 65(6):395-401 discussion401-2.

34. Parker RJ, Harris EF. Directions of orthodontics tooth movements associated with external apical root resorption of the maxillary central incisor. $A m$ J Orthod Dentofacial Orthop 1998 Dec; 114(6): 677-83.

35. Pizzo G, Licata ME, Guiglia R, Giuliana G. Root resorption and orthodontic treatment. Review of the literature. Minerva Stomatol 2007 Jan/Feb; 56(1-2): 31-44.

36. Rosenberg MN. An evaluation of an incidence and amount of apical root resorption and dilaceration occurring in orthodontically treated teeth having incompletely formed at the beginning of $B \operatorname{egg} g$ treatment. Am J Orthod Dentofacial Orthop 1972 May; 61(5): 524-25.
37. Rudolph CE. An evaluation of root resorption occurring during orthodontic treatment. J Dent Res 1940 Aug; 19(4):367-71.

38. Silva Filho OG, Hernandes R, Okada T. Efeitos induzidos pela expansão rápida da maxila sobre os pré-molares de ancoragem: estudo radiográfico. $\mathrm{O} r$ todontia 1994 set/dez; 27(3): 18-36.

39. Silva FILHO OG, Berreta EC, Cavassan AO, Capelozza FILHO L. Estimativa da reabsorção radicular em 50 casos ortodônticos bem finalizados. Ortodontia 1993 jan/abr; 26(1):24-35.

40. Taner T, Ciğer S, Sençift Y. Evaluation of apical root resorption following extraction therapy in subjects with class I and class II malocclusions. Eur J Orthod 1999 Oct; 21(5):491-6.

41. Ten Hoeve A, Mulie MR. The effect of anteroposterior incisor repositioning on the palatal cortex as studied with laminography. J Clin Orthod 1976 Nov; 10(11):804-22.

42. Viazis AD. Bioefficient therapy. J Clin Orthod, 1995 Sep; 29(9);552-68.

Recebido em: 26/09/2008

Aceito em: 4/05/2009 